\title{
PSP94, an upstream signaling mediator of prostasin found highly elevated in ovarian cancer
}

\author{
J-x Ma ${ }^{1,2,6}$, B-x Yan ${ }^{\star, 1,3,6}$, J Zhang ${ }^{3,4}$, B-H Jiang ${ }^{5}$, Y Guo ${ }^{1}$, H Riedel $^{1}$, MD Mueller ${ }^{1}$, SC Remick ${ }^{1}$ and JJ Yu ${ }^{1}$
}

Ovarian cancer is a leading cause of cancer death as diagnosis is frequently delayed to an advanced stage. Effective biomarkers and screening strategies for early detection are urgently needed. In the current study, we identify PSP94 as a key upstream factor in mediating prostasin (a protein previously reported to be overexpressed in ovarian cancer) signaling that regulates prostasin expression and action in ovarian cancer cells. PSP94 is overexpressed in ovarian cancer cell lines and patients, and is significantly correlated with prostasin levels. Signaling pathway analysis demonstrated that both PSP94 and prostasin, as potential upstream regulators of the Lin28b/Let-7 pathway, regulate Lin28b and its downstream partner Let-7 in ovarian cancer cells. Expression of PSP94 and prostasin show a strong correlation with the expression levels of Lin28b/Let-7 in ovarian cancer patients. Thus, PSP94/prostasin axis appears to be linked to the Lin28b/Let-7 loop, a well-known signaling mechanism in oncogenesis in general that is also altered in ovarian cancer. The findings suggest that PSP94 and PSP94/prostasin axis are key factors and potential therapeutic targets or early biomarkers for ovarian cancer.

Cell Death and Disease (2014) 5, e1407; doi:10.1038/cddis.2014.374; published online 4 September 2014

Epithelial ovarian cancer is the most lethal gynecologic cancer and the fourth leading cause of death among women in developed countries. If diagnosed at stage I with the malignancy confined to the ovary, the 5-year survival rates can reach up to $94 \%$. However, survival rates sharply decline to $28 \%$ for advanced stages at presentation. ${ }^{1}$ Insufficient biomarker(s) and the lack of an effective screening strategy for early detection result in $>80 \%$ of patients presenting with advanced disease at the time of diagnosis. Serum marker CA125 (cancer antigen 125) is clinically used in ovarian cancer screening, but it lacks the sensitivity and specificity to function independently in a test. ${ }^{2}$ A sensitive biomarker for accurate early detection of ovarian cancer is urgently needed.

Prostasin (protease serine 8 or PRSS8) is a trypsin-like serine peptidase expressed in epithelial cells and is found as a cell surface bound or secreted protein. Prostasin is altered in ovarian, prostate, breast and gastric cancers ${ }^{3-6}$ and has been proposed to inhibit cancer cell proliferation and invasion upon activation. $^{7}$ In particular, prostasin is overexpressed in ovarian cancer and may serve as a potential biomarker for early detection of ovarian cancer independently or in combination with CA125. ${ }^{4,8,9}$

Prostatic secretory protein 94 (PSP94) also termed microseminoprotein beta (MSMB) is the second most abundant protein in the semen of healthy men and is found in a variety of human tissues. ${ }^{10-12}$ The cellular levels of PSP94 gradually decreases with the progression of prostate cancer, suggesting that PSP94 may represent a promising target for cancer treatment and a potential biomarker for early detection of prostate cancer. ${ }^{13}$ In addition, genome-wide association studies and functional analysis of underlying MSMB gene correlate with polymorphism of the gene promoter region, resulting in altered MSMB expression with prostate cancer risk. ${ }^{14-17}$

MicroRNAs (miRNAs) regulate gene expression at posttranscriptional level in diversely biological functions such as cell proliferation, differentiation and apoptosis. ${ }^{18,19}$ The Let-7 family of miRNAs consists of more than 10 sequenceconserved members with similar functions across diverse species from worms to humans. ${ }^{20}$ Deregulation of Let-7 has been linked to many types of cancer and other diseases. ${ }^{20,21}$ An RNA-binding protein termed Lin28b was recently identified as a direct upstream inhibitor of Let-7 family signaling 22,23 with important functions in embryonic stem cells and embryonal carcinoma cells. ${ }^{24-27}$ Balanced signaling of Lin28b to Let-7 is critical; imbalance is linked to various diseases. ${ }^{28}$

Our recent studies demonstrated important roles and functional similarity of prostasin and PSP94 in ovarian cancer chemoresistance. ${ }^{29,30}$ In the current study, we report that prostasin expression is regulated by PSP94 and shares all examined downstream targets with PSP94 in ovarian cancer cells. PSP94 and prostasin are both overexpressed in strong correlation with Lin28b/Let-7 expression levels in tissues of ovarian cancer patients. As a result, PSP94 and the

\footnotetext{
${ }^{1}$ Department of Biochemistry, School of Medicine, Department of Basic Pharmaceutical Sciences, School of Pharmacy, and Mary Babb Randolph Cancer Center, Robert C. Byrd Health Sciences Center, West Virginia University, Morgantown, West Virginia, USA; ${ }^{2}$ Department of Molecular Microbiology and Immunology, Johns Hopkins Bloomberg School of Public Health, Johns Hopkins University, Baltimore, Maryland, USA; ${ }^{3}$ IcesnowYanyan Bioscience Association, Beijing, China; ${ }^{4}$ Beijing Animal Science Institute, Beijing, China and ${ }^{5}$ Department of Pathology, Anatomy and Cell Biology, Thomas Jefferson University, Philadelphia, Pennsylvania, USA.

${ }^{*}$ Corresponding author: B-x Yan, Department of Biochemistry, School of Medicine, Department of Basic Pharmaceutical Sciences, School of Pharmacy, and Mary Babb Randolph Cancer Center, Robert C. Byrd Health Sciences Center, West Virginia University, 1 Medical Center Drive, Morgantown, WV 26506 , USA. Tel: +1 304293 3124; Fax: +1 304293 4667; E-mail: bxueyan@ hotmail.com

${ }^{6}$ These authors contributed equally to this work.

Abbreviations: 0432 (Ovca432), human ovarian cancer parental cell line; O432-RP (Ovca432-RP), drug-resistant subline generated by repeatedly treated Ovca432 cells with paclitaxel

Received 11.6.14; revised 24.7.14; accepted 28.7.14; Edited by A Stephanou
} 
PSP94/prostasin axis may represent promising markers for early detection of ovarian cancer as well as potential therapeutic targets. This role may be extended to other types of cancers where either one or both components are altered.

\section{Results}

PSP94 regulates prostasin expression in ovarian cancer cells. PSP94 and prostasin both play important roles in chemoresistance and share similarities in their actions. ${ }^{29,30}$ To investigate their possible relationship, we examined PSP94 expression in prostasin overexpressing cells (O432-RP-pro-O) and prostasin level in PSP94 overexpressing cells (O432-RP-PSP-O). PSP94 expression was found unchanged in O432-RP-pro-O when compared to control cells (Figure 1, lane 3, top band), whereas prostasin level was found increased in O432-RP-PSP-O cells when compared to control cells O432-RP-C (Figure 1, lane 2, top band). These findings are consistent with a role of PSP94 as an upstream regulator for inducing prostasin expression, but not vice versa. We extended this observation to a different ovarian cancer cell line where, similarly, prostasin expression increased upon PSP94 overexpression (Supplementary Figure 1). We did not examine PSP94 expression since we were unable to produce prostasin overexpression, possibly due to the strong repression function of prostasin in ovarian cancer cells. $^{30}$

Prostasin is a downstream mediator of PSP94 in a shared signaling pathway. We previously showed that prostasin regulates downstream signaling of the CASP/ PAK2-p34 and MLCK/actin, JNK/c-Jun pathways. ${ }^{30}$ PSP94 may share these pathways with prostasin as an upstream mediator. We examined expression levels of various genes in CASP/PAK2-p34 and MLCK/actin, JNK/c-Jun pathways in PSP94 overexpressed cells O432-RP-PSP-O. All respective

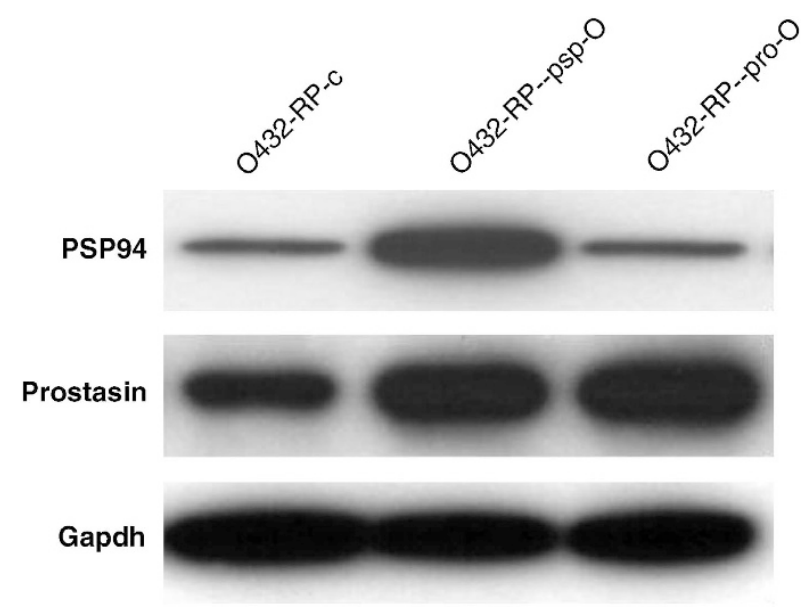

Figure 1 PSP94 regulates prostasin expression in ovarian cancer cells. Prostasin and PSP94 protein levels are shown in immunoblots with specific antibodies in prostasin overexpressed cells 0432-RP-pro-O and PSP94 overexpressed cells 0432-RP-PSP-O, respectively. Prostasin levels were increased in both cell models, but PSP94 level was increased only in O432-RP-PSP-O cells with no change in O432-RP-pro-O cells. O432-RP-C was used as a $\mathrm{pCl}-$ neo vector transfected control cell line genes were found to be altered in O432-RP-PSP-O cells when compared to the control O432-RP-C (Figure 2a), and showed the same pattern as in prostasin overexpressed cells (O432-RP-pro-O when compared to the control). ${ }^{30}$ Our findings suggest shared signaling mechanisms between PSP94 and prostasin in CASP/PAK2-p34 and MLCK/actin, JNK/c-Jun pathways. PSP94 has been shown to regulate the Lin28/Let-7 loop in ovarian cancer cells. ${ }^{29}$ To investigate whether prostasin participates in this mechanism together with PSP94, we examined Lin28b and Let-7 expression levels in prostasin overexpressed cells O432-RP-pro-O. Lin28b level was increased, whereas Let-7 miRNA level decreased in O432-RP-pro-O cells compared to the control (Figures $2 \mathrm{~b}$ and $\mathrm{c}$ ). Based on our observations, PSP94 appears to function as an upstream mediator of prostasin and both proteins share all tested downstream targets. PSP94 regulates prostasin expression, but prostasin does not appear to affect PSP94 level.

Prostasin and PSP94 have similar roles in chemoresistance in ovarian cancer $^{29,30}$ and share downstream targets. Our data suggest a role for prostasin as a key downstream target of PSP94 in the development of chemoresistance. To investigate this hypothesis, we studied O432-RP-PSP-O-pro-D cells obtained by prostasin-siRNA transfection of O432-RPPSP-O cells (Figure 2d). Both cell lines were treated with paclitaxel and cell survival was evaluated. As shown in Figure $2 \mathrm{e}$, the survival of O432-RP-PSP-O-pro-D cells increased in a dose-dependent manner that indicates greater resistance to paclitaxel treatment when compared to O432-RP-PSP-O cells. Knockdown prostasin in O432-RPPSP-O cells appeared to interfere with PSP94 action in chemoresistance by disconnecting PSP94 from its downstream targets. These data demonstrate that PSP94 is dependent on prostasin function in the development of chemoresistance in our experimental model.

PSP94 or the PSP94/prostasin axis is overexpressed in ovarian cancer cell lines and ovarian cancer patients. Prostasin is overexpressed in ovarian cancer patients and several ovarian cancer cell lines ${ }^{4}$ and PSP94 show a comparable expression pattern. When we compared PSP94 protein levels in normal and several ovarian cancer cell lines, we found high or modest levels of PSP94 expression in all cancer cell lines but not detectable in normal ovarian cells (Figure 3a). We also quantified PSP94 and prostasin mRNA expression in ovarian cancer tissues. As shown in Figure 3b, PSP94 mRNA levels are significantly higher (about four-fold, on average) in tumor tissues of ovarian cancer patients (at all stages) than in normal specimens. We also compared mRNA levels in early and late stages of the disease (stages 1 and 2 were defined as early stage, versus stages 3,4 and 5 as late stage). We observed that PSP94 levels are four to five-fold higher in early stage samples and slightly elevated above control level in the late stages. A similar pattern of prostasin expression was also observed in these samples (Supplementary Figure 2).

Correlation analysis of PSP94 and prostasin expression showed significant overlap in ovarian cancer tissues (Figure 3c). Among 13 matched samples from ovarian cancer 


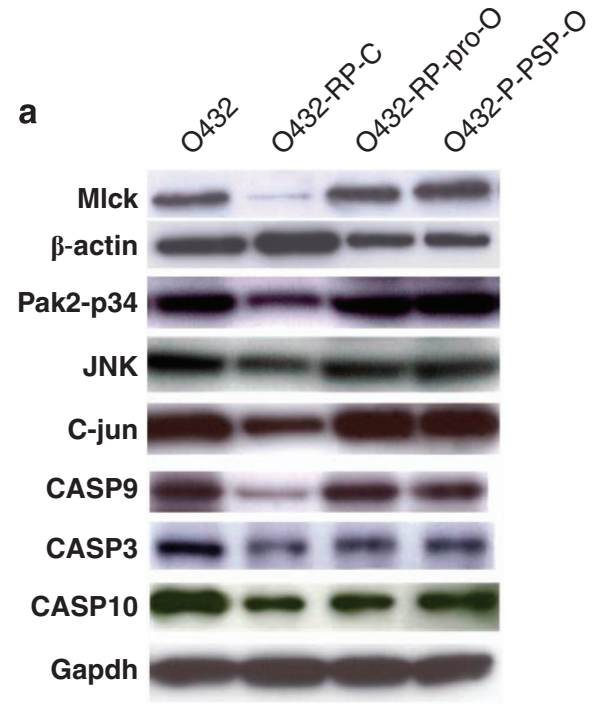

C
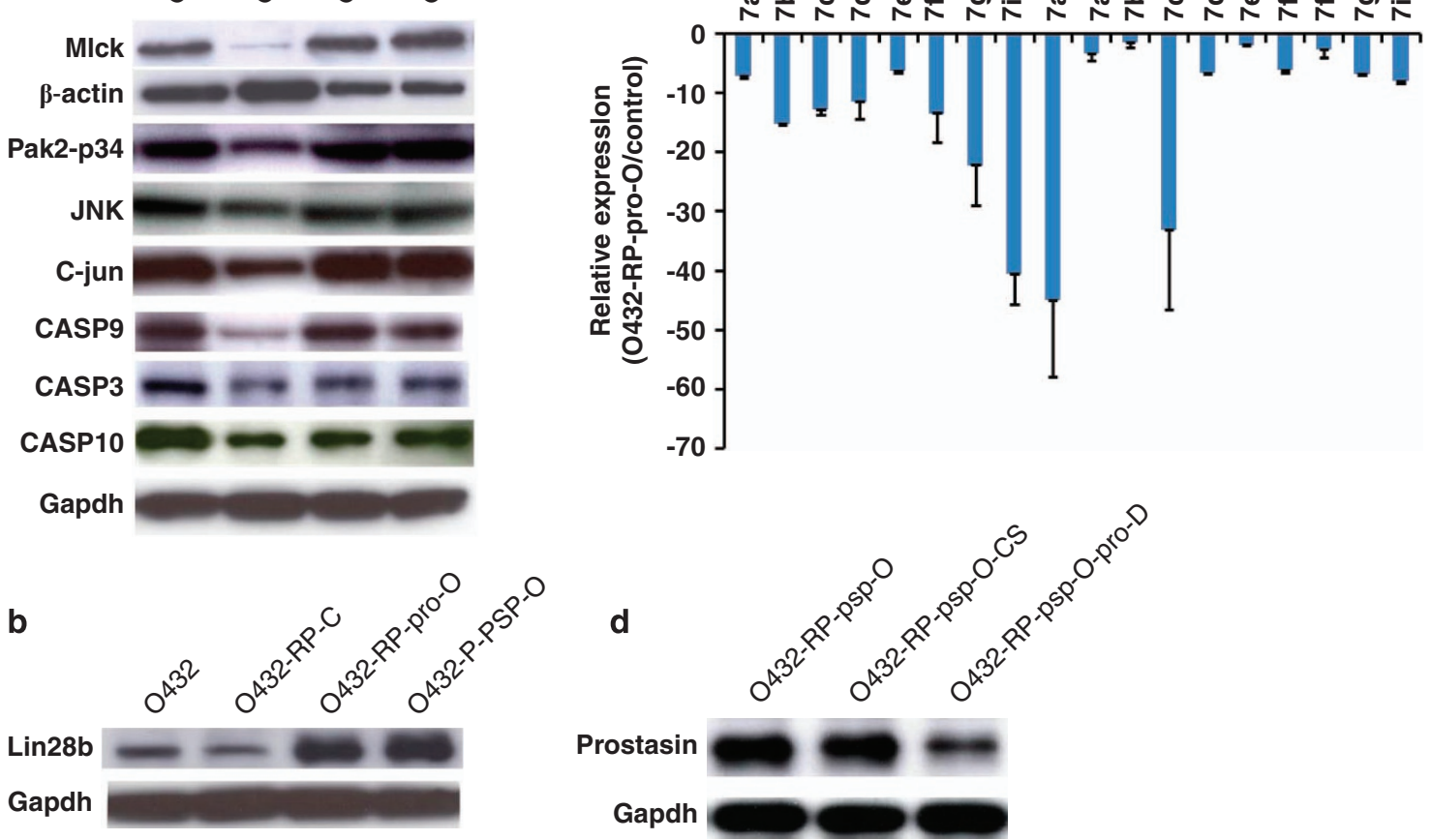

e

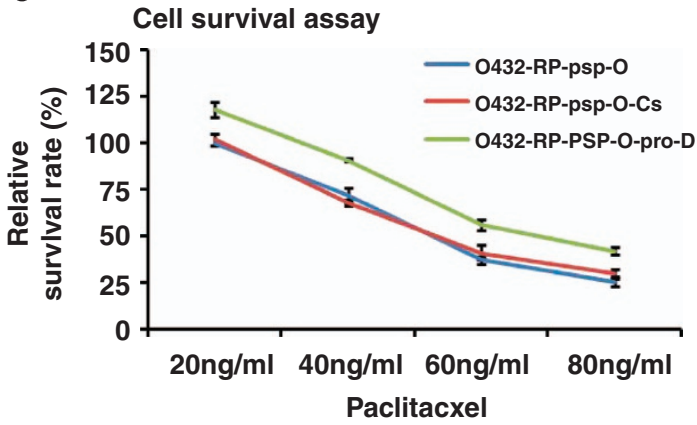

Figure 2 Prostasin is a downstream mediator of PSP94 in a shared signaling pathway. (a) CASP/PAK2-p34 signaling and further downstream JNK/c-Jun and MLCK/actin signaling are regulated by PSP94. Expression levels of CASPs, PAK2-p34, JNK, c-Jun, MLCK and actin were examined in PSP94 overexpressed O432-RP-PSP-0 cells, which show the same pattern as prostasin overexpressed cells 0432-RP-pro-O. Overexpression of prostasin in 0432-RP-pro-0 cells upregulates CASP/PAK2-p34 and further downstream JNK/C-Jun and MLCK/actin signaling molecules (lanes 1,2 and 3) as previously reported, ${ }^{30}$ indicating that PSP94 and prostasin share the same downstream targets. ( $\mathbf{b}$ and $\mathbf{c}$ ) Prostasin regulates Lin28b/Let-7 loop in ovarian cancer cells. Expression levels of Lin28b and Let-7 microRNA were examined in prostasin overexpressed cells (O432-RP-pro-0) and the same expression pattern is seen as in PSP94 overexpressed O432-RP-PSP-O cells. Upregulation of Lin28b by overexpression of PSP94 (O432-RP-PSP-O; lanes 1, 2 and 4) is consistent with our previous report, ${ }^{29}$ indicating that PSP94 and prostasin share the same downstream targets. (d) ProstasinsiRNA knockdown. Prostasin protein levels are decreased in 0432-RP-PSP-O-pro-D knockdown cells compared to O432-RP-PSP-O (mock transfection control) or O432-RPPSP-O-CS (siRNA transfection control). (e) Prostasin-siRNA knockdown in 0432-RP-PSP-0 cells appeared to interfere with PSP94 action in chemoresistance. Cells were plated at $10-20 \%$ confluence, treated with paclitaxel at different concentrations for $24 \mathrm{~h}$, and then cultured in normal medium for $7-10$ days. Cell survival was evaluated. Relative survival rates of each cell line are shown

patients (tumor tissue and normal ovary tissue from the same patient) 11 were seen PSP94 expression increased in tumors compared to normal tissue ( $\sim 85 \%$; a $C t$ value $>36$ considered as positive expression and vice versa; Figure $3 \mathrm{~d}$ ).

PSP94 protein is secreted in various human tissues including breast and ovaries. ${ }^{12}$ To evaluate whether PSP94 could serve as a serum biomarker for early detection of ovarian cancer, we examined PSP94 protein level in the blood of normal and ovarian cancer patients. In ovarian cancer patients (mixed stages), PSP94 levels were $35.7 \pm 21.7 \mathrm{ng} / \mathrm{ml}$, which is significantly higher compared to normal controls $(14.6 \pm 12.7 \mathrm{ng} / \mathrm{ml}$; Table $1 ; P<0.001)$, confirming that PSP94 is overexpressed in ovarian cancer patients.

PSP94 and prostasin regulate the Lin28/Let-7 loop in ovarian cancer cells and show correlation with Lin28/ Let-7 expression in cancer patients. Expression of Lin28b and Let-7 is altered in ovarian cancer. ${ }^{31,32}$ Our previous 
a

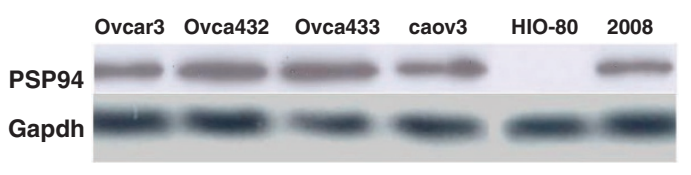

b

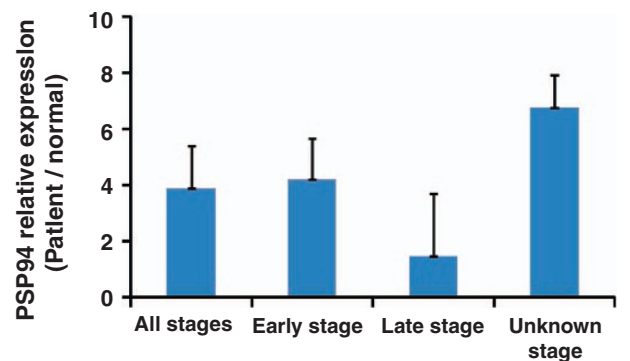

C

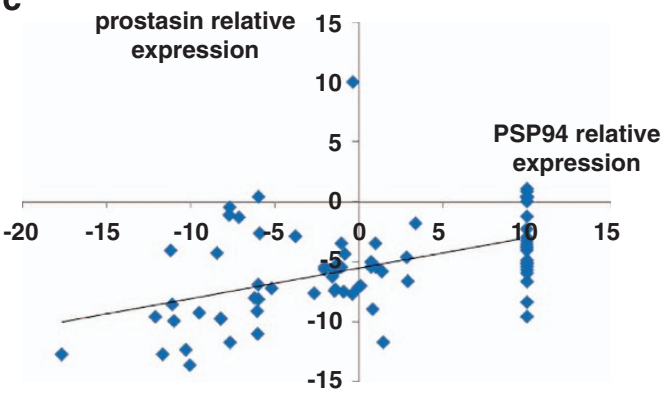

d

Patients

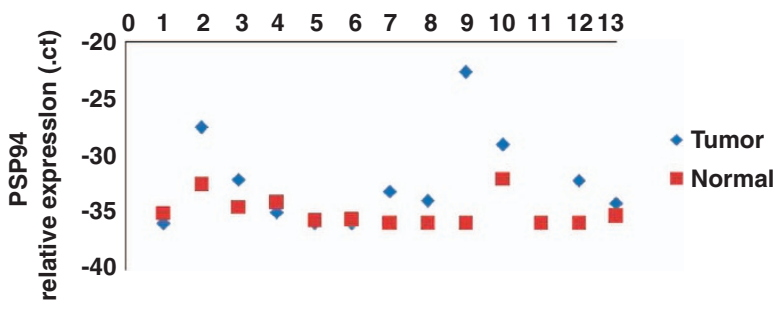

Figure 3 PSP94 expression levels are elevated in ovarian cancer cells and tumor tissues. (a) PSP94 expression levels by immunobloting with specific antibodies in human ovarian normal and cancer cell lines. PSP94 protein levels are shown in all ovarian cancer cell lines Ovca432, Ovca433, Ovcar3, Caov3 and 2008, but no PSP94 expression is seen in normal ovarian surface epithelial (HIO-80) cells. (b) PSP94 is overexpressed in ovarian cancer patients. PSP94 mRNA levels were measured in tumor tissues of ovarian cancer patients and compared among different stages by real-time QPCR analysis. Relative levels of PSP94 in patients (early, late, unknown and all stages) were compared to normal controls. In this study, early stage $n=43$, late stage $n=14$, unknown stage $n=19$ and normal control, $n=35$. (Stages 1 and 2 were defined as early stage and stages 3,4 and 5 as late stage.) $P<0.05$. Mean \pm S.D. are given, and the $P$-values were calculated using the two-sided Student's $t$-test. (c) Correlation between PSP94 and prostasin in ovarian cancer patients. (d) Comparison of PSP94 levels in 13 patients with matched ovarian tumor and normal tissues. PSP94 mRNA levels were examined by real-time qPCR analysis. The $\mathrm{Ct}$ value of each matched samples (normal and tumor tissues of same patient) was compared. $\mathrm{Ct}=36 \mathrm{was}$ set as non-detectable level

studies and current investigation demonstrate that both PSP94 and prostasin regulate Lin28b/Let-7 expression in ovarian cancer cells $0432-\mathrm{RP}^{29}$ (Figure 2). Consequently, the PSP94/prostasin axis may act as an upstream mediator of the Lin28b/Let-7 loop, altering its expression in ovarian cancer. We further examined the expression levels of Lin28b and Let-7 in ovarian cancer tissues by qPCR. As shown in Figure 4, PSP94 and prostasin showed strong positive correlation with Lin28b ( $R=0.33$ and 0.19 , respectively), but negative correlation with Let-7 expression in ovarian cancer tissues $(R=-0.26$ and -0.27 , respectively). These findings suggest a close functional relationship between the PSP94/prostasin axis and the Lin28b/Let-7 loop in the development of ovarian cancer.

\section{Discussion}

Despite dramatic advances in recent decades in understanding basic cancer mechanisms, ovarian cancer is still a deadly disease with a five-year survival rate of $<50 \% .{ }^{33}$ In particular, the lack of effective biomarkers for early screening results in $>80 \%$ of patients presenting with advanced disease at the time of diagnosis. In this study, we show that PSP94 is overexpressed in ovarian cancer patients and cell lines and functions as an upstream mediator of prostasin. Since PSP94 expression is particularly elevated in early-stage patients (stages1 and 2), and only somewhat in late-stage patients (stages 3 and 4), when compared to normal controls, PSP94 is a possible biomarker for early detection of ovarian cancer.

Other studies report that prostasin is overexpressed in ovarian cancer and in combination with CA125 provides high sensitivity (92\%) and specificity (94\%) to identify cancer patients, but low sensitivity when used as an individual marker. ${ }^{4}$ We propose PSP94 as a more advantageous biomarker for earlier detection of ovarian cancer, compared to prostasin. PSP94 is a secreted protein, ${ }^{10}$ whereas prostasin is a secreted and membrane protein (glycosylphosphatidylinositol-anchored ${ }^{5}$ ), with functional differences between the two proteins. ${ }^{10,30}$ Prostasin appears to be implicated in a much wider spectrum of physiological and pathophysiological conditions than PSP94 and has important roles in epidermal barrier function, skin phenotype, embryonic viability and blood pressure. ${ }^{34-38}$ In contrast, known functions of PSP94 are relatively narrow in prostate cancer development and ovarian cancer chemoresistance. Although PSP94 is a promising biomarker for ovarian cancer detection and treatment, further studies are needed. Large patient samples are required to determine sensitivity and specificity and functional studies are necessary to investigate PSP94 (and its peptide derivative PCK3145) as potential therapeutic targets.

Our findings show that PSP94 is an upstream mediator of prostasin in ovarian cancer. PSP94 and prostasin are overexpressed in ovarian cancer patients, and PSP94/prostasin signaling may play an essential role in ovarian cancer 
development. PSP94 and prostasin levels are both decreased in prostate cancer; this is consistent with our preliminary findings that PSP94 regulates prostasin expression in prostate cancer cells and likely serves as an upstream signaling mediator of prostasin in prostate cancer development. We expect that PSP94/prostasin signaling may be important in oncogenesis in general, not just limited to ovarian cancer (Figure 5), and plays an important role in ovarian cancer chemoresistance. ${ }^{29,30}$ Notably, PSP94/prostasin signaling appears to have an opposite role in ovarian cancer compared to prostate cancer, as PSP94 and prostasin are overexpressed in ovarian cancer, ${ }^{4}$ while their expression levels are decreased in prostate cancer. ${ }^{3,13}$ Prostasin expression is also altered in breast and gastric cancers ${ }^{5,6}$ and it may be meaningful to investigate the corresponding PSP94 expression in those cancers.

In our study, PSP94 is an upstream signaling mediator of prostasin in ovarian cancer cells, and both share all investigated downstream targets; however, prostasin may have other upstream regulators and downstream signals that are independent of PSP94 (Figure 5). This is suggested by some functional differences that we observed between PSP94 and prostasin in ovarian cancer cells. For example, recombinant PSP94 or PSP94-derived peptide PCK3145

Table 1 PSP94 levels in the blood of normal and ovarian cancer patients

\begin{tabular}{lcccccccc}
\hline & $\begin{array}{c}\text { Mean } \\
(\mathbf{n g} / \mathbf{m l})\end{array}$ & S.D. Range & $\boldsymbol{n}$ & Age & $\boldsymbol{P}$ & \\
\hline $\begin{array}{l}\text { Cancer } \\
\text { patients }\end{array}$ & 35.7 & 21.7 & $0-78$ & 19 & $31-69$ & 0.0007 & $\begin{array}{c}\text { Mixed } \\
\text { stages }\end{array}$ \\
Normal & 14.6 & 12.7 & $0-61$ & 28 & $29-78$ & & \\
\hline
\end{tabular}
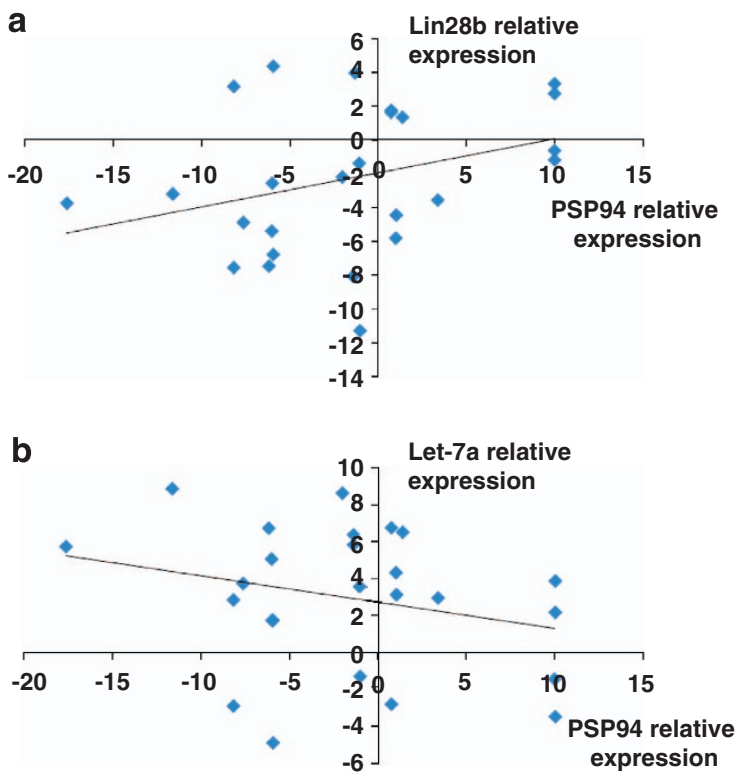

represses some ovarian cancer cells, in contrast to recombinant prostasin. Overexpression of prostasin in ovarian cancer cells greatly induces cell death and represses cell survival, whereas overexpression of PSP94 in ovarian cancer cells does not significantly affect cell death. ${ }^{29,30}$ Thus, further studies on differences between these two genes in ovarian cancer are needed to comprehensively understand their role in oncogenesis.

Lin28b was recently identified as an RNA-binding protein and direct upstream mediator and inhibitor of Let-7 family. ${ }^{22,23}$ Alteration of Let-7 or Lin28b has been linked to many types of cancers and other diseases, and the Lin28/Let-7 loop is seen as a crucial signaling circuit in oncogenesis. ${ }^{20,21,32,39-41}$ Particularly, upregulation of Lin28b and altered Let-7 are associated with advanced disease and reduced patient survival in ovarian cancer $25,31,42,43$ and a Lin28b polymorphism has been linked to susceptibility to epithelial ovarian cancer. $^{32}$ Our findings show that PSP94 and prostasin regulate Lin28b and Let-7 expression in ovarian cancer cells and their expression appear to be strongly correlated in ovarian cancer patients (Figure 4). Our data suggest that alteration of Lin28b and Let-7 expression in ovarian cancer may partially result from overexpression of PSP94 and prostasin. It is widely accepted that Lin28b overexpression contributes to tumorigenesis by repressing tumor suppressor Let-7 expression in ovarian and other cancers, ${ }^{20,21,32,39-41}$ which is consistent with our view that the PSP94/prostasin pathway may play a key role in the development of several cancer types (Figure 5). Our current findings suggest a potential link between Lin28b/Let-7 signaling and the PSP94/ prostasin pathway in oncogenesis in general. The Lin28/Let-7 pathway is also believed to play a critical role in stem cell development. ${ }^{18,22,23}$ Thus, our new findings point to a
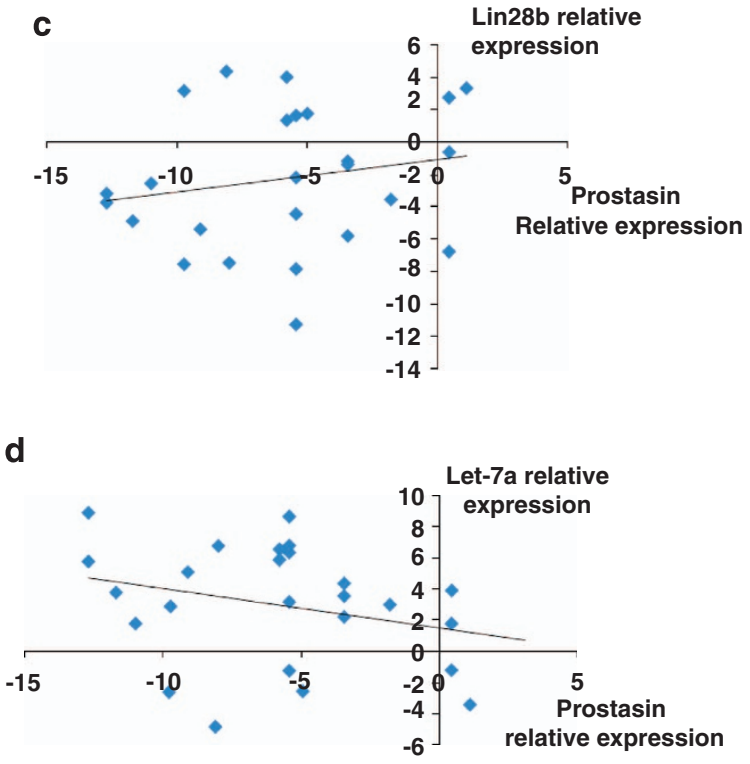

Figure 4 Correlation between PSP94/prostasin signaling and the Lin28b/Let-7 loop in ovarian cancer. (a) PSP94 expression shows positive correlation with Lin28b expression in ovarian cancer patients. The prediction line is shown: $r=0.33, n=25$. (b) PSP94 expression shows an inverse correlation with Let-7a expression in ovarian cancer patients. The prediction line is shown: $r=0.26, n=25$. (c) Prostasin expression shows positive correlation with Lin28b expression in ovarian cancer patients. The prediction line is shown: $r=0.19, n=25$. (d) Prostasin expression shows an inverse correlation with Let-7a expression in ovarian cancer patients. The prediction line is shown: $r=0.27, n=25$ 


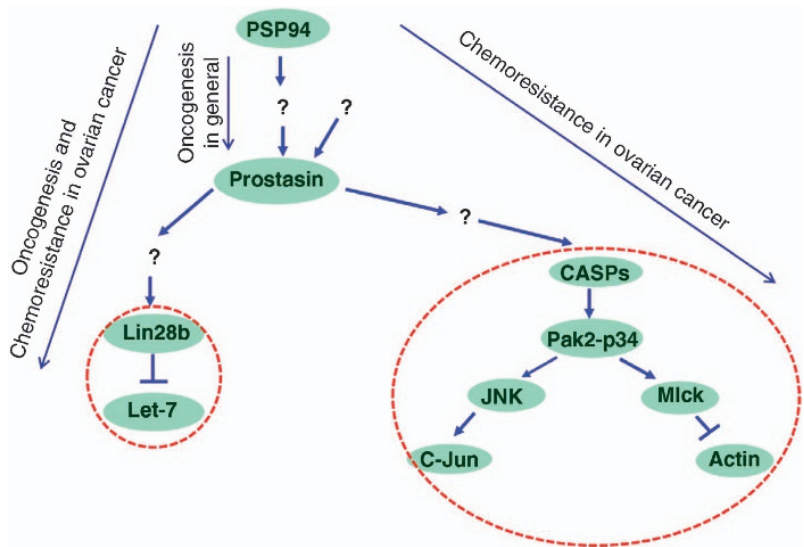

Figure 5 Proposed model of a PSP94/prostasin-regulated signaling network in oncogenesis and chemoresistance. The PSP94-prostasin axis may play key roles in oncogenesis in general. Signaling of PSP94/prostasin and Lin28b/Let-7 plays critical roles in ovarian cancer development and chemoresistance. Signaling of PSP94/prostasin and downstream subpathways of the CASP/PAK2-p34, JNK/ c-Jun and MLCK/actin plays important roles in chemoresistance in ovarian cancer

connection between PSP94/prostasin signaling and the Lin28b/Let-7 loop in oncogenesis and possibly stem cell development.

In summary, our findings show that PSP94 is an upstream signaling mediator of prostasin; PSP94 or PSP94/prostasin signaling is activated and induced in ovarian cancer, and is connected to the Lin28/Let-7 pathway.

\section{Materials and Methods}

Human samples and ethics statement. Frozen GOG (Gynecologic Oncology Group) samples (54 tumors and 35 normal ovarian tissues including 13 pairs of matched samples) from patients with advanced ovarian cancer were obtained from the Cooperative Human Tissue Network (CHTN), Pediatric Division, Children's Hospital, Columbus, Ohio, USA. Additional 25 frozen ovarian tumor tissues and plasma samples (19 ovarian cancer and 28 normal controls) were obtained from Heilongjiang Nongken Hospital (Haerbin, Heilongjiang, China). The tumor specimens were collected at primary surgery, freshly frozen in liquid nitrogen and stored at $-80^{\circ} \mathrm{C}$ until RNA/DNA extraction. Plasma samples were first stored at $-20^{\circ} \mathrm{C}$ and then transferred to $-80^{\circ} \mathrm{C}$ before analysis. Samples had been stripped off all patient identifiers before shipping to ensure that the presented molecular laboratory data cannot be linked with any patient. All samples were evaluated by pathologists.

Cell lines and cell culture. All cell lines were propagated as an adherent monolayer at $37^{\circ} \mathrm{C}$ in a humidified atmosphere of $5 \% \mathrm{CO}_{2}$ supplemented with $10 \%$ heat-inactivated FBS, $100 \mu \mathrm{g} / \mathrm{ml}$ penicillin and $100 \mu \mathrm{g} / \mathrm{ml}$ streptomycin. The HOSE cell line HIO-80 was maintained in a 1:1 mixture of medium M199 and MCDB-105 (Invitrogen, Life Technologies, Inc., Carlsbad, CA, USA). All other cell lines were maintained in MEM (Invitrogen). Normal ovarian HIO-80 cells were provided by Dr. Andrew Godwin at the University of Kansas. Ovarian cancer 2008 cells were provided by Dr. Aikou Okamoto (Jikei University, Japan); Ovca432 was provided by Dr. Liu T from Chinese Academy of Sciences; Ovca433, Ovcar3 and Caov3 were purchased from ATCC; Ovca432-RP, 0432-RP-pro-0, 0432-RP-PSP-O and $0432-\mathrm{RP}-\mathrm{C}$ were previously generated in our laboratory. ${ }^{29,30}$

RNA extraction and cDNA generation. Total RNA from GOG samples was purified by hot phenol/chloroform extraction as previously reported. ${ }^{44}$ Through reverse transcription, using the Super Script Preamplification System (Life Technologies, Inc.), cDNA was generated with oligo-dT primers from $5 \mu \mathrm{g}$ of total RNA per sample (Life Technologies, Inc.). Total RNA of rest tumor tissues was prepared with TRIzol reagents (Life Technologies, Inc.). cDNA was synthesized using Taqman Reverse Transcription (PE Applied Biosystems, Foster City, CA, USA) from $1 \mu \mathrm{g}$ of total RNA. CDNAs were used for gene expression profiling.
Real-time reverse transcription quantitative PCR. Real-time quantitative PCR on an ABI PRISM 7900 or 7500 instrument (PE Applied Biosystems) was performed according to the manufacturer's instructions and as previously described. ${ }^{29} \mathrm{PCR}$ cycling conditions were set at $50^{\circ} \mathrm{C}$ for $2 \mathrm{~min} ; 95^{\circ} \mathrm{C}$ for $10 \mathrm{~min}$; 40 cycles for the melting $\left(95^{\circ} \mathrm{C}\right.$ for $\left.15 \mathrm{~s}\right)$ and annealing/extension $\left(60^{\circ} \mathrm{C}\right.$ for $\left.1 \mathrm{~min}\right)$ steps. qPCR reactions for each template were carried out in triplicate on 96-well plates. Comparative $\mathrm{Ct}$ (PE Applied Biosystems) determined relative expression in each sample using $18 \mathrm{~S}$ rRNA as endogenous control.

Western blot. As previously described, ${ }^{29}$ cells were rinsed twice with PBS and solubilized in lysis buffer ( $150 \mathrm{mM}$ sodium chloride; $50 \mathrm{mM}$ Tris hydrochloride, $\mathrm{pH}$ 7.5; $1 \%$ glycerol; $1 \%$ Nonidet P-40 substitute solution (Sigma-Aldrich, Inc., St. Louis, MO, USA)). Equal amounts of total protein were loaded and separated by SDS-PAGE. Proteins were transferred to a polyvinylidenedifluoride membrane, which was blocked in 5\% skimmed milk, and probed with specific (primary) antibody, followed by treatment with secondary antibody conjugated to horseradish peroxidase $(1: 5000)$. Proteins were visualized by (enhanced) chemiluminescence detection (Pierce Biotechnology) and exposure to X-ray film. Beta-actin or GAPDH proteins were visualized by normal chemiluminescence detection (Pierce Biotechnology) and exposed to less-sensitive X-ray film, which may result in a relatively weaker signal. All antibodies used in this study were obtained from Abnova (Taipei, Taiwan, China) or Abcam (Cambridge, MA, USA).

siRNA and cDNA transfection. Cells were transfected with prostasin or PSP94 cDNA containing vectors using Lipofectamine 2000 (Invitrogen) according the manufacturer's instructions. ${ }^{29,30}$ The parental $\mathrm{pCl}$-neo vector served as a negative control and stably expressing cells were obtained by Geneticin (Life Technologies, Inc.) selection. For siRNA experiments, mock transfections and non-targeting siRNA were used as negative controls. All siRNA experiments used two alternative siRNAs targeting the same gene to ensure knockdown, and all experiments were repeated at least three times. Cells were treated for $72 \mathrm{~h}$ to allow for maximum knockdown, and then lysed for immunoblot analysis, RNA preparation or other assays. siRNA oligos were purchased from Ambion (Austin, TX, USA).

miRNA isolation and quantification. Total RNA was isolated by TRIzol extraction (Invitrogen). Two micrograms of total RNA were reverse-transcribed into cDNA using the QuantiMir RT Kit (System Biosciences, Mountain View, CA, USA) to convert all small RNAs simultaneously into detectable cDNAs for real-time PCR. miRNA levels were quantified with an ABI PRISM 7900 instrument (PE Applied Biosystems) and U6 served as an internal control.

Cell survival assay. Cell survival was evaluated as previously described. ${ }^{29}$ Cells were counted and plated into culture dishes at $\sim 10-20 \%$ confluence on the day before treatment. Paclitaxel or PBS as control was added for $20 \mathrm{~h}$ and then removed. Cells were recovered and continuously propagated in normal medium for 10 days. To quantify final cell numbers, cells were stained with $0.25 \%$ crystal violet $/ 20 \%$ ethanol and counted; or the proliferation rate was measured using a Cell Proliferation Assay kit (Promega, Madison, WI, USA) according to the manufacturer's instruction. Briefly, MTS/PMS solution (at a final concentration of $333 \mu \mathrm{g} / \mathrm{ml}$ MTS and $25 \mu \mathrm{M}$ PMS) was added to each well, and cells were incubated for $2-3 \mathrm{~h}$ at $37^{\circ} \mathrm{C}$. The absorbance was determined at $490 \mathrm{~nm}$ using a 96-well plate ELISA reader. Culture medium was used as a background control. The experiments were repeated at least three times.

ELISA and PSP94 measurements. The plasma PSP94 concentration was determined using a PSP94 ELISA Kit (Sanbo Inc., Beijing, China); $100 \mu \mathrm{l}$ of standard or plasma samples were loaded to 96-well plates and incubated for $1 \mathrm{~h}$ at $37^{\circ} \mathrm{C}$ with $50 \mu \mathrm{l}$ of HRP conjugate. After rinsing, $50 \mu \mathrm{l}$ of substrate $\mathrm{A}$ and $50 \mu \mathrm{l}$ of substrate $\mathrm{B}$ were added to each well and incubated for $15 \mathrm{~min}$ at $37^{\circ} \mathrm{C} ; 50 \mu \mathrm{l}$ of Stop Solution (Sanbo Inc, Beijing, China) was added before the absorbance at $450 \mathrm{~nm}$ was determined with a 96-well plate ELISA reader.

Statistical analysis. The Student's $t$-test and correlation analysis for Windows were used for statistical analysis with significance defined as $P<0.05$. All statistical tests and corresponding $P$-values were two-sided.

\section{Conflict of Interest}

The authors declare no conflict of interest. 
Acknowledgements. We would like to thank Dr. Andrew K Godwin at The University of Kansas Medical Center for providing the immortalized human ovarian surface epithelial (HIO-80) cells for our investigation. We acknowledge support for this study from the Molecular Medicine Core Facility, Mary Babb Randolph Cancer Center, West Virginia University, Morgantown, WV, USA and the IcesnowYanyan Bioscience Association, Beijing, China.

1. Jemal A, Siegel R, Xu J, Ward E. Cancer statistics, 2010. CA Cancer J Clin 2010; 60: 277-300.

2. Nossov V, Amneus M, Su F, Lang J, Janco JM, Reddy ST et al. The early detection of ovarian cancer: from traditional methods to proteomics. Can we really do better than serum CA-125? Am J Obstet Gynecol 2008; 199: 215-223.

3. Chen LM, Hodge GB, Guarda LA, Welch JL, Greenberg NM, Chai KX. Down-regulation of prostasin serine protease: a potential invasion suppressor in prostate cancer. Prostate 2001; 48: 93-103

4. Mok SC, Chao J, Skates S, Wong K, Yiu GK, Muto MG et al. Prostasin, a potential serum marker for ovarian cancer: identification through microarray technology. J Natl Cancer Inst 2001; 93: 1458-1464.

5. Chen LM, Chai KX. Prostasin serine protease inhibits breast cancer invasiveness and is transcriptionally regulated by promoter DNA methylation. Int J Cancer 2002; 97: 323-329.

6. Sakashita K, Mimori K, Tanaka F, Tahara K, Inoue H, Sawada T et al. Clinical significance of low expression of Prostasin mRNA in human gastric cancer. J Surg Oncol 2008; 98 : $559-564$

7. Lopez-Otin C, Matrisian LM. Emerging roles of proteases in tumour suppression. Nat Rev Cancer 2007; 7: 800-808

8. Lu KH, Patterson AP, Wang L, Marquez RT, Atkinson EN, Baggerly KA et al. Selection of potential markers for epithelial ovarian cancer with gene expression arrays and recursive descent partition analysis. Clin Cancer Res 2004; 10: 3291-3300.

9. Costa FP, Batista EL Jr, Zelmanowicz A, Svedman C, Devenz G, Alves S et al. Prostasin, a potential tumor marker in ovarian cancer-a pilot study. Clinics (Sao Paulo) 2009; 64: 641-644

10. Lilja $\mathrm{H}$, Abrahamsson PA. Three predominant proteins secreted by the human prostate gland. Prostate 1988; 12: 29-38.

11. Weiber H, Andersson C, Murne A, Rannevik G, Lindstrom C, Lilja $\mathrm{H}$ et al. Beta microseminoprotein is not a prostate-specific protein. Its identification in mucous glands and secretions. Am J Pathol 1990; 137: 593-603.

12. Baijal-Gupta M, Clarke MW, Finkelman MA, McLachlin CM, Han VK. Prostatic secretory protein (PSP94) expression in human female reproductive tissues, breast and in endometrial cancer cell lines. J Endocrinol 2000; 165: 425-433.

13. Whitaker HC, Warren AY, Eeles R, Kote-Jarai Z, Neal DE. The potential value of microseminoprotein-beta as a prostate cancer biomarker and therapeutic target. Prostate 2010; 70: 333-340.

14. Thomas $G$, Jacobs KB, Yeager M, Kraft $P$, Wacholder S, Orr N et al. Multiple loci identified in a genome-wide association study of prostate cancer. Nat Genet 2008; 40: 310-315.

15. Eeles RA, Kote-Jarai Z, Giles GG, Olama AA, Guy M, Jugurnauth SK et al. Multiple newly identified loci associated with prostate cancer susceptibility. Nat Genet 2008; 40 : 316-321.

16. Lou H, Yeager M, Li H, Bosquet JG, Hayes RB, Orr N et al. Fine mapping and functional analysis of a common variant in MSMB on chromosome 10q11.2 associated with prostate cancer susceptibility. Proc Natl Acad Sci USA 2009; 106: 7933-7938.

17. Xu B, Wang J, Tong N, Mi Y, Min Z, Tao J et al. A functional polymorphism in MSMB gene promoter is associated with prostate cancer risk and serum MSMB expression. Prostate 70: $1146-1152$

18. Bussing I, Slack FJ, Grosshans H. let-7 microRNAs in development, stem cells and cancer. Trends Mol Med 2008; 14: 400-409.

19. Schickel R, Boyerinas B, Park SM, Peter ME. MicroRNAs: key players in the immune system, differentiation, tumorigenesis and cell death. Oncogene 2008; 27: 5959-5974.

20. Pasquinelli AE, Reinhart BJ, Slack F, Martindale MQ, Kuroda MI, Maller B et al. Conservation of the sequence and temporal expression of let-7 heterochronic regulatory RNA. Nature 2000; 408: 86-89.

21. Boyerinas B, Park SM, Hau A, Murmann AE, Peter ME. The role of let-7 in cell differentiation and cancer. Endocr Relat Cancer 2010; 17: F19-F36.

22. Piskounova E, Polytarchou C, Thornton JE, LaPierre RJ, Pothoulakis C, Hagan JP et al Lin28A and Lin28B inhibit let-7 microRNA biogenesis by distinct mechanisms. Cell 2011 147: 1066-1079.

23. Viswanathan SR, Daley GQ, Gregory RI. Selective blockade of microRNA processing by Lin28. Science 2008; 320: 97-100.

24. Guo $Y$, Chen $Y$, Ito $H$, Watanabe $A$, Ge $X$, Kodama $T$ et al. Identification and characterization of lin-28 homolog B (LIN28B) in human hepatocellular carcinoma. Gene 2006; 384: 51-61.
25. Viswanathan SR, Powers JT, Einhorn W, Hoshida Y, Ng TL, Toffanin S et al. Lin28 promotes transformation and is associated with advanced human malignancies. Nat Genet 2009; 41: 843-848.

26. King CE, Wang L, Winograd R, Madison BB, Mongroo PS, Johnstone CN et al. LIN28B fosters colon cancer migration, invasion and transformation through let-7-dependent and -independent mechanisms. Oncogene 2011; 30: 4185-4193.

27. Hagan JP, Piskounova E, Gregory RI. Lin28 recruits the TUTase Zcchc11 to inhibit let-7 maturation in mouse embryonic stem cells. Nat Struct Mol Biol 2009; 16: 1021-1025.

28. Ji J, Wang XW. A Yin-Yang balancing act of the lin28/let-7 link in tumorigenesis. J Hepatol 2010; 53: 974-975

29. Yan BX, Ma JX, Zhang J, Guo Y, Riedel H, Mueller MD et al. PSP94 contributes to chemoresistance and its peptide derivative PCK3145 represses tumor growth in ovarian cancer. Oncogene 2013; e-pub ahead of print 4 November 2013; doi:10.1038/ onc. 2013.466

30. Yan BX, Ma JX, Zhang J, Guo Y, Mueller MD, Remick SC et al. Prostasin may contribute to chemoresistance, repress cancer cells in ovarian cancer, and is involved in the signaling pathways of CASP/PAK2-p34/actin. Cell Death Dis 2014; 5: e995.

31. Lu L, Katsaros D, Shaverdashvili K, Qian B, Wu Y, de la Longrais IA et al. Pluripotent factor lin-28 and its homologue lin-28b in epithelial ovarian cancer and their associations with disease outcomes and expression of let-7a and IGF-II. Eur J Cancer 2009; 45: 2212-2218.

32. Permuth-Wey J, Kim D, Tsai YY, Lin HY, Chen YA, Barnholtz-Sloan J et al. LIN28B polymorphisms influence susceptibility to epithelial ovarian cancer. Cancer Res 2011; 71: 3896-3903.

33. Siegel R, Naishadham D, Jemal A. Cancer statistics, 2012. CA Cancer J Clin 2012; 62: 10-29.

34. Rickert KW, Kelley P, Byrne NJ, Diehl RE, Hall DL, Montalvo AM et al. Structure of human prostasin, a target for the regulation of hypertension. J Biol Chem 2008; 283: 34864-34872

35. Planes $\mathrm{C}$, Randrianarison $\mathrm{NH}$, Charles RP, Frateschi S, Cluzeaud F, Vuagniaux G et al. ENaC-mediated alveolar fluid clearance and lung fluid balance depend on the channel-activating protease 1. EMBO Mol Med 2010; 2: 26-37.

36. Li NF, Zhang JH, Chang JH, Yang J, Wang HM, Zhou L et al. Association of genetic variations of the prostasin gene with essential hypertension in the Xinjiang Kazakh population. Chin Med J (Engl) 2011; 124: 2107-2112.

37. Frateschi S, Keppner A, Malsure S, Iwaszkiewicz J, Sergi C, Merillat AM et al. Mutations of the serine protease CAP1/Prss8 lead to reduced embryonic viability, skin defects, and decreased ENaC activity. Am J Pathol 2012; 181: 605-615.

38. Leyvraz C, Charles RP, Rubera I, Guitard M, Rotman S, Breiden B et al. The epidermal barrier function is dependent on the serine protease CAP1/Prss8. J Cell Biol 2005; 170: 487-496

39. Madison BB, Liu Q, Zhong X, Hahn CM, Lin N, Emmett MJ et al. LIN28B promotes growth and tumorigenesis of the intestinal epithelium via Let-7. Genes Dev 2013: 27: 2233-2245.

40. Zhou J, Ng SB, Chng WJ. LIN28/LIN28B: an emerging oncogenic driver in cancer stem cells. Int J Biochem Cell Biol 2013; 45: 973-978.

41. Alajez NM, Shi W, Wong D, Lenarduzzi M, Waldron J, Weinreb I et al. Lin28b promotes head and neck cancer progression via modulation of the insulin-like growth factor survival pathway. Oncotarget 2012; 3: 1641-1652.

42. Faggad A, Budczies J, Tchernitsa O, Darb-Esfahani S, Sehouli J, Muller BM et al. Prognostic significance of Dicer expression in ovarian cancer-link to global microRNA changes and oestrogen receptor expression. J Pathol 2010; 220: 382-391.

43. Merritt WM, Lin YG, Han LY, Kamat AA, Spannuth WA, Schmandt R et al. Dicer, Drosha, and outcomes in patients with ovarian cancer. N Engl J Med 2008; 359: 2641-2650.

44. Yu J, Dabholkar M, Bennett W, Welsh J, Mu C, Bostickbruton F et al. Platinum-sensitive and platinum-resistant ovarian cancer tissues show differences in the relationships between mRNA levels of p53, ERCC1 and XPA. Int J Oncol 1996; 8: 313-317.

(2) (1) $\odot$ Cell Death and Disease is an open-access journal published by Nature Publishing Group. This work is licensed under a Creative Commons Attribution-NonCommercialNoDerivs 3.0 Unported License. The images or other third party material in this article are included in the article's Creative Commons license, unless indicated otherwise in the credit line; if the material is not included under the Creative Commons license, users will need to obtain permission from the license holder to reproduce the material. To view a copy of this license, visit http://creativecommons.org/ licenses/by-nc-nd/3.0/ 